\title{
NEEUC: Energy Efficient Unequal Clustering Algorithm for Wireless Sensor Network
}

\author{
Prof. N R Wankhade ${ }^{1}$, Dr. D N Choudhari ${ }^{2}$, Dr. V M Thakre ${ }^{3}$ \\ Associate Professor, Department of Computer Engineering, LGNSCOE, Pune University, Nashik, India ${ }^{1}$ \\ Professor, Dept of Computer Science Engineering, Sant Gadge Baba Amravati University, Amravati, Maharashtra, India ${ }^{2}$ \\ Professor \& Head Department of Computer Science Engineering, Sant Gadge Baba Amravati University, Amravati, \\ Maharashtra, India ${ }^{3}$
}

\begin{abstract}
Energy saving is a critical issue in Wireless Sensor Network as they have limited amount of energy. In WSN Sensor node closer to the sink will exhaust their limited energy more rapidly than other sensor nodes, since they will have to forward huge data during multi hop transmission to the base station. Therefore network lifetime will be reduced because of hotspot problem. To cope up with the network lifetime, in this paper, Novel Energy Efficient Unequal Clustering algorithm for clustered wireless sensor network is proposed, the decision of selecting cluster heads by the sink is based on the residual energy and distance to the base station, Besides, the cluster head also selects the shortest path to reach the sink. Simulation results show that our approach enhanced the performance than traditional routing algorithms, such as LEACH \& EEUC.
\end{abstract}

Index Terms: Residual energy, Unequal clustering, Routing, Network lifetime, Hot Spot problem.

\section{INTRODUCTION}

\section{A. Detail Problem Definition}

Wireless sensor networks are of tiny, battery powered sensor nodes with limited processing power and capabilities. To overcome this problem and to achieve higher energy efficiency, sensor nodes are grouped into clusters, where each cluster heads collects all the data and process them before being sent to the base station. To achieve network scalability, clustering [1] is the best solution. The potential applications of sensor networks are highly varied, such as environmental monitoring, and battlefield surveillance. Sensors in such a network are equipped with sensing, data processing and wireless communication capabilities. Distinguished from traditional wireless networks, sensor networks are characterized by severe power, computation, and memory constraints. Due to limited and non-rechargeable energy provision, the energy resource of sensor networks should be managed wisely to extend the lifetime [2] of sensors. We consider a network of energy-constrained sensors that are deployed over a geographic area for monitoring the environment. Each sensor periodically produces information as it monitors its vicinity. The basic operation in such a network is the systematic gathering and transmission of sensed data to a base station for further processing. In order to achieve high energy efficiency and increase the network scalability, sensor nodes can be organized into clusters. Data collected from sensors are sent to the cluster head first, and then forwarded to the base station.

\section{B Need of Proposed System}

In this paper, we propose and evaluate a Novel Energy Efficient Unequal Clustering Routing (NEEUC) protocol for mitigating the hot spot problem in wireless sensor networks. In LEACH[1] cluster heads are selected randomly therefore the distribution of cluster head is uneven, as result energy consumption is unbalanced. HEED [2] select candidate cluster head through the remaining energy and generate the cluster head according to minimum reachable power. More delay and more cost generated because of iterative communication between node in cluster head selection because of equal cluster size. EEUC[3] balance the energy consumption but it applies only to the area where nodes are deployed evenly. It also produces isolated nodes as cluster head selection is probabilistic. In this paper we proposed Novel Energy Efficient Unequal Clustering Algorithm (NEEUC).In NEEUC cluster closer to the base station are smaller and consume less energy during transmission. Our algorithm consists of two parts, one is an Unequal Clustering algorithm for topology management, and the other is a multi-hop routing protocol for inter cluster communication. The main contribution of the paper is that we provide the first unequal cluster based routing protocol to mitigate the hot spot problem and thus prolong the network lifetime. NEEUC is a self-organized competition based algorithm, where cluster heads are selected based on local information (i.e., the residual energy of neighboring nodes).

This paper is organized as follows: Section II describes most recent research within the area of clustering algorithm is wireless sensor network; Section III describe network model; Section IV presents NEEUC algorithm; Section $\mathrm{V}$ analyses performance of NEEUC algorithm. 


\section{RELATED WORK}

There has been substantial research in the area of routing in wireless sensor networks. Many equal and unequal clustering algorithms have been proposed to prolong the network lifetime [3]. We review some of the most relevant papers on unequal clustering. An unequal clustering model was first proposed in (Heinzelman et al 2000) [1] based on unequal clustering. In multi hop network unequal clustering was $30-40 \%$ better as compared to equal clustering like LEACH. LEACH uses randomized rotation of cluster heads to distribute energy consumption over all nodes in the network. And uses single hop communication. An energy-aware variant of LEACH is proposed in which the nodes with higher energy are more likely to become cluster heads. However, the underlying routing protocol is assumed to be able to propagate node residual energy through the network. The authors analytically determine the optimum number of cluster heads. HEED [2] introduces a variable known as cluster radius which defines the transmission power to be used for intra-cluster broadcast. The initial probability for each node to become a tentative cluster head depends on its residual energy, and final heads are selected according to the intra-cluster communication cost. HEED terminates within a constant number of iterations, and achieves fairly uniform distribution of cluster heads across the network. HEED does not make any assumption like network size or density. EECS $[3,4]$ introduces a cluster head competitive algorithm without message exchange iterations. It extends LEACH and HEED by choosing cluster heads with more residual energy. It also achieves a decent distribution of cluster heads. While the clustering problem has been extensively explored, researchers have only recently started to study the strategies for balancing the workload among cluster heads while considering the inter-cluster traffic. In single hop sensor networks, cluster heads use direct communication to reach the base station, and the problem of unbalanced energy consumption among cluster heads arises. Cluster heads farther away from the base station have heavier energy burden due to the long-haul communication links. Consequently, they will die earlier. In EECS a distance based cluster formation method is proposed to produce clusters of unequal sizes. Clusters farther away from the base station have smaller sizes, thus some energy could be preserved for long-haul data transmission to the base station. On the other hand, the hot spot problem arises when multi hop routing is adopted when cluster heads deliver their data to the base station. Heinzelman first investigate an unequal clustering model for balancing the energy consumption of cluster heads in multi hop sensor networks. The work focuses on a Heterogeneous network where cluster heads (super nodes) are deterministically deployed at pre-computed locations. Through both theoretical and experimental analyses, the authors show that unequal clustering could be beneficial, especially for heavy traffic applications.

In the energy efficient unequal clustering (EEUC) $[7,8]$ algorithm ( $\mathrm{Li}$ et al., 2005), the authors propose another unequal clustering algorithm where nodes join clusters of unequal size. However, according to (Gong et al., 2008), EEUC may produce lone nodes since the cluster head election is probabilistic. Zhao et al. propose an unequal layered clustering approach for large scale wireless sensor network (ULCA) (Zhao and Wang, 2010)

In (Heinzelman et al., 2000), the Lower Energy Adaptive Clustering Hierarchy (LEACH) protocol is presented. The algorithm elects cluster heads solely based on probability. No residual energy is taken into account. Moreover, cluster heads use the single hop communication model to forward packets to the base station. A refined version of LEACH can be found in (Xuhui et al., 2009). The lifetime of the sensor net- works is maximized by first forming unequal clusters, and then a new threshold algorithm, based on residual energy, is used to elect cluster heads.

In (Pin et al., 2010), the authors propose IEEUC, which is similar to the study in EEUC (Li et al., 2005), since it creates unequal sized clusters as they are further away from the base station, to mitigate the hotspot problem. The main difference between IEEUC [9.10] and EEUC lies in the competition radius calculation. IEEUC uses the node degree factor, which is based on the number of hops to the base station, to calculate the competition radius. In EEUC, even clusters equidistant from the BS may have different number of member nodes, either too many or too few. On the other hand in IEEUC, we do not have this problem.

The hot spot problem addressed in this paper is due to the many-to-one multi hop data forwarding pattern on the cluster head backbones. Researchers have proposed several methods to mitigate this kind of hot spot problem. Perillo et al. [15] analyse two such strategies. Although the network lifetime can be improved by using a more intelligent transmission power control policy that balances the energy consumption of each node, they conclude that it cannot solve the hot spot problem on its own.

They also investigate the effectiveness and cost efficiency of using a heterogeneous clustering hierarchy to mitigate the hot spot problem.

\section{NETWORK MODEL}

The network consists of a set of sensors which are uniformly deployed over a field to continuously monitor the environment. We make some assumptions about the sensor nodes and the network model.

- All the nodes are alike and each node is assigned with a unique identifier (ID).

- Base station is located at the center of the monitor field. Sensors and the base station are all stationary after deployment.

- A node can compute the approximate distance to another node based on the received signal strength.

- Node can transmit in different power levels to achieve different communication range.

We use a simplified model presented in [1]. Either the free space (d2 power loss) or the multi-path (d4 power loss) 
Vol. 5, Issue 12, December 2016

channel is used based on the distance between the ordinary nodes as its members. Similar to LEACH, the transmitter and the receiver. The energy spent for operation of this algorithm is divided into rounds. The task transmitting h-bit packet over distance $\mathrm{d}$ is:

$$
\operatorname{ETX}(\mathrm{h}, \mathrm{d})\left\{\begin{array}{l}
h E_{e l e c}+h \varepsilon_{f g} d^{2} \ldots \ldots . d \leq d_{0} \\
h E_{e l e c}+h \varepsilon_{m p} d^{4} \ldots \ldots d>d_{0}
\end{array}\right.
$$

Where $\mathrm{d} 0$ is the critical distance beyond which multi-path channel mode is used. Energy spent for receiving h-bit packet is

$$
\operatorname{ERX}(\mathrm{h}, \mathrm{d})=\text { hEelec }
$$

All the cluster head can always aggregate the data coming from its member into a single length-fixed packet.

\section{NEEUC ROUTING ALGORITHM}

\section{A.Assumption}

1. Sensors uniformly deploy over a sensor field

2. Sensors and a BS are all stationary

3. Sensors can use power control

4. A node can compute the

5. Approximate distance to another node based on the received signal strength

The key idea of NEEUC is to mitigate the Hotspot problem.

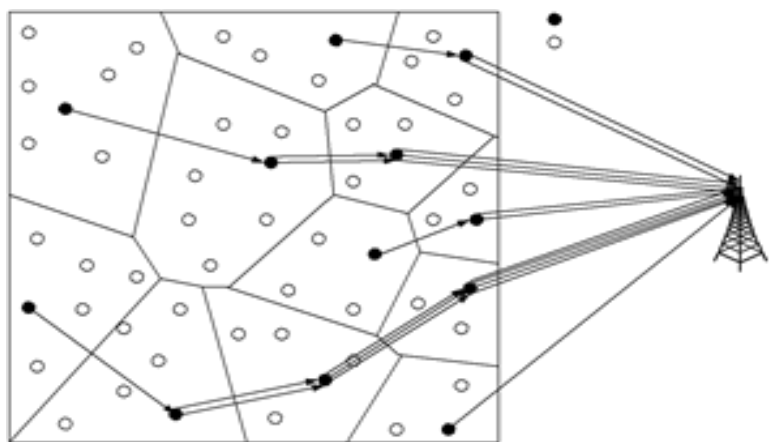

Fig.1 Unequal clustering mechanism

We introduce a novel energy efficient unequal clustering protocol for hierarchical routing, called NEEUC. It organizes the network into clusters of unequal sizes. By decreasing the number of nodes in clusters with higher relay load near the base station, we can maintain more uniform energy consumption among cluster heads in the long run.

B. Novel Unequal clustering algorithm

The Algorithm includes the following phases

a). Design of network structure

b) Determine communication radius

c) Clustering mechanisms

d) Multi hops transmission.

Clustering a wireless sensor network means partitioning its nodes into clusters, each one with a cluster head and some of being a cluster head is rotated among sensors in each round to distribute the energy consumption across the network. NEEUC is a distributed cluster head competitive algorithm, where the cluster head selection is primarily based on the residual energy and distance to the base station. The algorithm for determine scope of each layer is follows.

a) Design Network Structure

The BS broadcasts a "hello" message to all nodes.

1. Each node computes the distance to the BS based on signal strength and determine layer using following formula.

$$
\begin{aligned}
& \text { Upper }=\mathrm{dmin}+\mathrm{i} * \mathrm{dmin} / \mathrm{m} \quad, \quad \mathrm{i}=1,2,-----\mathrm{n} \\
& \text { Lower }=\mathrm{dmin}+(\mathrm{i}-1) * \mathrm{dmax}-\mathrm{dmin} / \mathrm{m}, \mathrm{i}=1,2,----\mathrm{n}
\end{aligned}
$$

Where dmin \& dmax -shortest and longest distance between node and base station.

Assume distance between node and base station is greater than lower boundary \& less than upper boundary then node belongs to layer $i$.

b) Determine cluster Radius

In this phase cluster radius of each node computed based on distance to the base station and residual energy.

$$
\begin{gathered}
\text { Radius }=\left[\varepsilon _ { 1 } \left(1-\mathrm{c} * \mathrm{dmax}-\mathrm{d}\left(\mathrm{S}_{\mathrm{i}}, \mathrm{BS}\right) / \mathrm{dmax}-\right.\right. \\
\left.\mathrm{dmin})+\varepsilon 2 * \mathrm{E}_{\mathrm{Th}} / \text { Einit }\right] * \text { Ro }
\end{gathered}
$$

Where dmin \& $\mathrm{d}_{\max }$-shortest and longest distance between node and base station.

$\mathrm{d}\left(\mathrm{S}_{\mathrm{i}}, \mathrm{BS}\right)$ - distance from node I to base station.

$\mathrm{C}, \varepsilon_{1}, \varepsilon 2$ - constant between $0-1$

Ro- system setting of cluster radius.

Radius varies as residual energy \& distance between node to $\mathrm{BS}$ varies.

c) Clustering algorithm

Step-1 node generate random number between 0-1

If Random-no $<\mathrm{T}$ then node becomes candidate node.

Step-2 Candidate node send message to its radius calculated in above formula.

Step-3 if node i can receive a message(id,Ri,RE) from node $\mathrm{j} \&$ distance between both nodes is less than any competition radius of each other, then node $i$ store node $j$ in $\mathrm{S}_{\text {ct.. }}$ Each tentative $\mathrm{CH}$ maintains a set $\mathrm{S}_{\mathrm{CH}}$ of its "adjacent" tentative $\mathrm{CH}$.

Where $\mathrm{Ri}$ - radius RE-residual energy

Step-4 Node i compare its own energy with the energy of each node in $\mathrm{S}_{\mathrm{ct} . . .}$ If the energy of node $\mathrm{i}>$ any node stored in $\mathrm{S}_{\mathrm{ct.} .}$ then node becomes $\mathrm{CH}$ and release a message Finish_Elect_Meassage within its cluster radius.

Step-5 According to information within Compate_Head_ Meassage non $\mathrm{CH}$ node join with $\mathrm{CH}$.If non $\mathrm{CH}$ received more message then use larger signal strength. 
Vol. 5, Issue 12, December 2016

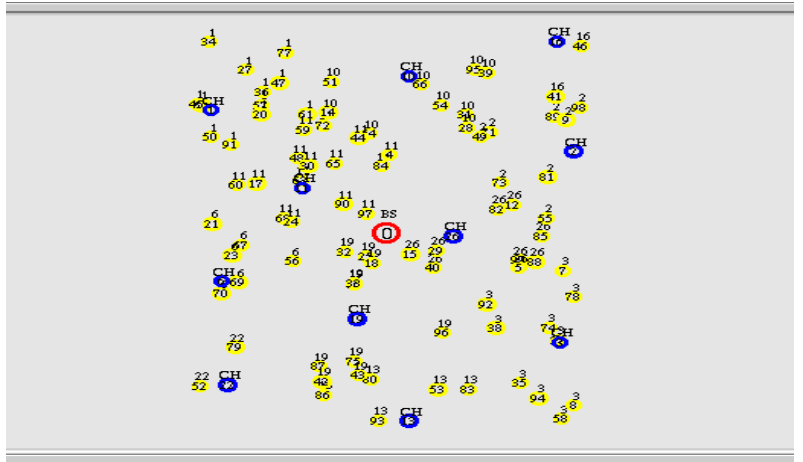

Fig. $2 \mathrm{CH}$ distribution, where no. of node $=100$

\section{d) Multi Hop communications}

In single-hop communication the furthest member nodes or $\mathrm{CHs}$ tend to deplete their battery energy faster than other nodes in a network. In other words, in single-hop where data packets are directly transmitted to the $\mathrm{CH}$ or the BS without any relay, the nodes located farther away have higher energy burden due to long-range communication, and these nodes may die out first. To overcome this problem, we have used multi-hop communication between member nodes and their respective $\mathrm{CH}$ and also between $\mathrm{CHs}$ and the $\mathrm{BS}$.

We use the data transmission network by a directed weighted graph $G=\{V, E\}$,, where $V$ is a set of nodes and $E$ is a set of edges. Let us assume vi and vj are two nodes in the graph. For the edge $\mathrm{e}=(\mathrm{vi}, \mathrm{vj}), \mathrm{w}(\mathrm{e})=\mathrm{wij}$, which indicates the weight of e. Here, wij represents the wasting energy of node vi which is given in the following equation if node vi transfers data to node

vj:Wij $=\{\mathrm{L} \times($ Eelec $+\epsilon \mathrm{fs} \times \mathrm{d} 2 \mathrm{ij}), \mathrm{L} \times($ Eelec $+\epsilon \mathrm{mp} \times \mathrm{d} 4 \mathrm{ij})$,

if $\mathrm{dij} \leq \mathrm{d} 0$, if $\mathrm{dij} \geq \mathrm{d} 0$, where dij is the distance between vi and vj. Similarly, if vj is the second hop node chosen by another node and vt is the BS, then wjt represents the wasting energy of node vj which is given by the following: $\mathrm{Wjt}=\{2 \times \mathrm{L} \times($ Eelec $+\epsilon \mathrm{fs} \times \mathrm{d} 2 \mathrm{ij}), 2 \times \mathrm{L} \times($ Eelec $+\epsilon \mathrm{mp} \times \mathrm{d} 4 \mathrm{ij})$, if $\mathrm{dij}$ $\leq \mathrm{d} 0$, if $\mathrm{dij} \geq \mathrm{d} 0$, because it includes a receiving consumption. Therefore, the shortest path weight, also called distance, from vi and vt, denoted d(vs, vt) or dst, is the minimum weight of all possible directed paths with origin vs and destination vt Our algorithm uses the greedy approach to solve the single source shortest problem. It repeatedly selects from the unselected vertices, vertex $\mathrm{v}$ nearest to source $\mathrm{s}$ and declares the distance to be the actual shortest distance from $\mathrm{s}$ to $\mathrm{v}$. The edges of $\mathrm{v}$ are then checked to see if their destination can be reached by $\mathrm{v}$ followed by the relevant outgoing edges.

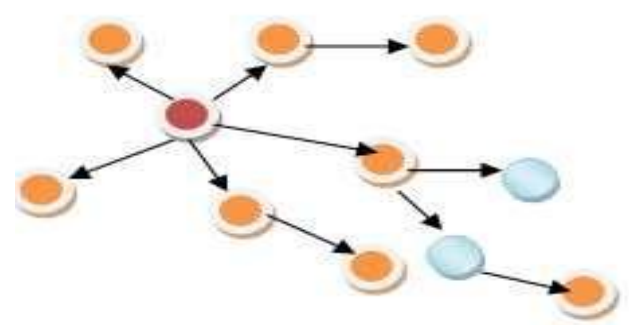

Fig.3. Multipath route construction

\section{RESULTS AND DISCUSSION}

In this section performance of proposed algorithm is evaluated. Network Simulator is used for simulation. We have calculated value of Rmax using novel equation to enhanced energy efficiency. The simulation parameter's are given in Table I.

TABLE I Simulation parameters

\begin{tabular}{|l|c|}
\hline Simulation parameter & Unit \\
\hline No of sensor nodes & 150 \\
\hline Network Region & $1000 * 1000$ \\
\hline Initial Energy & 100 Joules \\
\hline Packet length & 4000 bits \\
\hline Simulation time & $200 \mathrm{~s}$ \\
\hline Channel Capacity & $2 \mathrm{Mbps}$ \\
\hline
\end{tabular}

We observe that the propose algorithm has been able to calculate the best communication range for $\mathrm{CH}$ to optimize the energy usage. Energy consumed by CH's and normal nodes in LEACH and EEUC is greater than NEEUC. In fig 4 throughput in NEEUC is better than LEACH and EEUC, we also observed in fig 5 data delivery ratio is the ratio of number of packet received successfully and total number of packet transmitted. Also in in fig 6 good utilization of resources i.e control overhead in NEEUC. In fig 7 ratio of packet drop in LEACH is greater than NEEUC algorithm, also we observed in fig 8 that node delay in LEACH is greater than NEEUC algorithm. We observed that node overhead in LEACH is more than NEEUC algorithm.

TABLE II Cluster Head communication range

\begin{tabular}{|c|c|c|c|}
\hline $\begin{array}{c}\text { Cluster } \\
\text { Number }\end{array}$ & $\begin{array}{c}\text { Communications } \\
\text { radius }\end{array}$ & $\begin{array}{c}\text { Residual } \\
\text { Energy }\end{array}$ & $\begin{array}{c}\text { Distance to } \\
\text { the BS }\end{array}$ \\
\hline 1 & $13 \mathrm{~m}$ & $0.3382 \mathrm{~J}$ & $23.79 \mathrm{~m}$ \\
2 & $13 \mathrm{~m}$ & $0.3395 \mathrm{~J}$ & $11.01 \mathrm{~m}$ \\
3 & $12.75 \mathrm{~m}$ & $0.334 \mathrm{~J}$ & $12.57 \mathrm{~m}$ \\
4 & $11.76 \mathrm{~m}$ & $0.3163 \mathrm{~J}$ & $21.73 \mathrm{~m}$ \\
5 & $11.07 \mathrm{~m}$ & $0.2850 \mathrm{~J}$ & $26.11 \mathrm{~m}$ \\
6 & $11.03 \mathrm{~m}$ & $0.2996 \mathrm{~J}$ & $17.15 \mathrm{~m}$ \\
7 & $10.98 \mathrm{~m}$ & $0.3062 \mathrm{~J}$ & $23.89 \mathrm{~m}$ \\
8 & $10.17 \mathrm{~m}$ & $0.2767 \mathrm{~J}$ & $26.15 \mathrm{~m}$ \\
9 & $10.08 \mathrm{~m}$ & $0.2876 \mathrm{~J}$ & $27.36 \mathrm{~m}$ \\
10 & $9.84 \mathrm{~m}$ & $0.2876 \mathrm{~J}$ & $27.80 \mathrm{~m}$ \\
11 & $9.82 \mathrm{~m}$ & $0.2621 \mathrm{~J}$ & $28.45 \mathrm{~m}$ \\
12 & $9.81 \mathrm{~m}$ & $0.2662 \mathrm{~J}$ & $14.14 \mathrm{~m}$ \\
13 & $8.46 \mathrm{~m}$ & $0.2331 \mathrm{~J}$ & $32.21 \mathrm{~m}$ \\
\hline
\end{tabular}

In fig 4 the average rate of successful packets delivered which will be represented in packet per round. It shows the increase in the throughput of NEEUC against the number of nodes. The path which needs minimum energy is chosen to transmit and receive the data. Hence the lifetime of the nodes may be increased and hence reduce the control overhead which leads to increase in the throughput. 
Vol. 5, Issue 12, December 2016

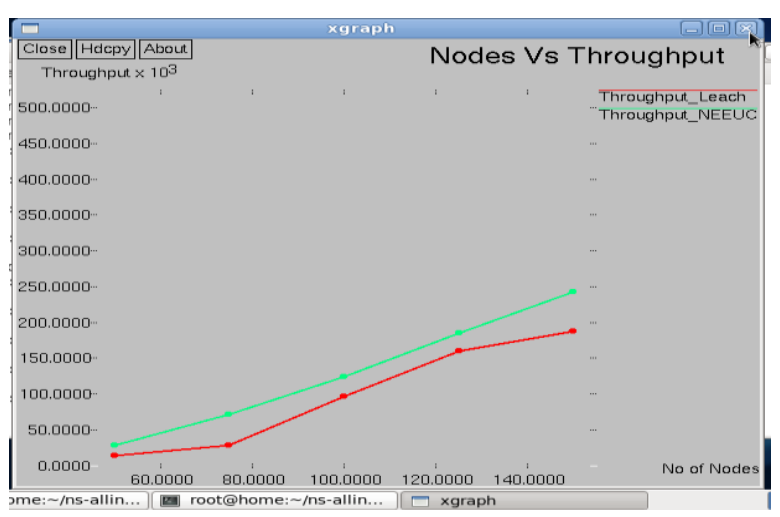

Fig. 4 Node Vs Throughput

In fig 5 the results show that the Packet delivery ratio of the proposed NEEUC is significantly increased when compared with LEACH. The increase is due to the selection of the path which needs minimum energy to transmit the data which in turn reduces the number of control packets to find out the neighbour, if any of the nodes are dead due to the scarcity of power.

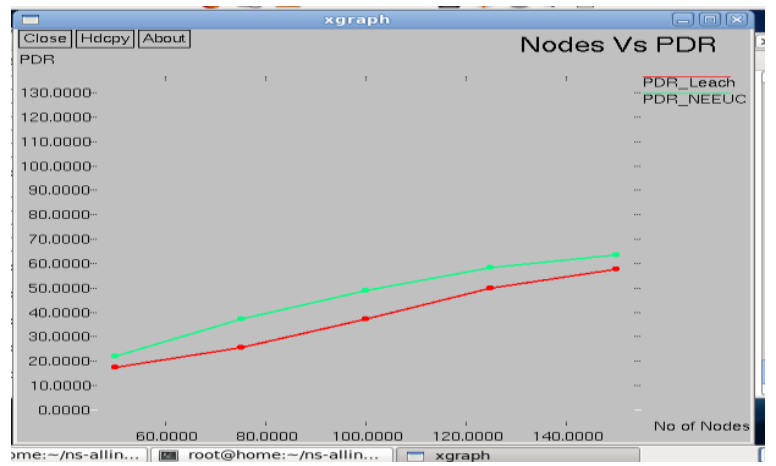

Fig. 5 Node Vs packet Delivery Ratio

NEEUC make use of more utilization of memory, bandwidth and other resources for achieving good result

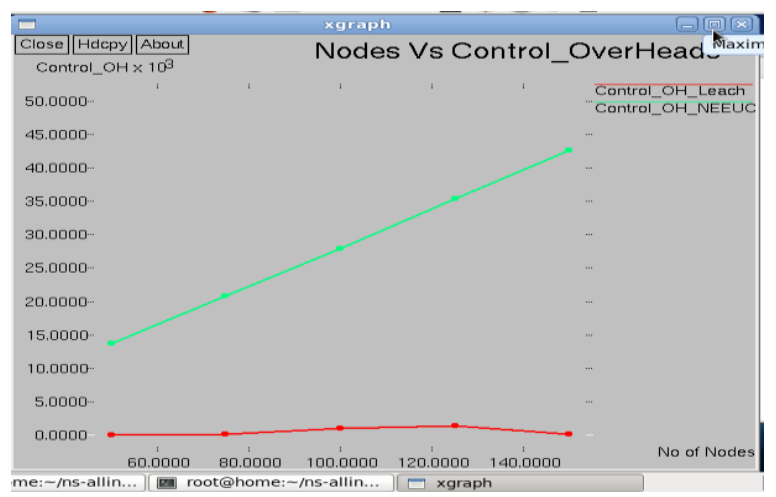

Fig. 6 Node Vs Control Overhead

We have also studied the impact of packet loss on the energy consumption of the entire network. In NEEUC by choosing best path can significantly improve the energy lost by each node when communicating. In figure 7 shows the minimizing dropping packet in NEEUC as compared to $\mathrm{LEACH}$.

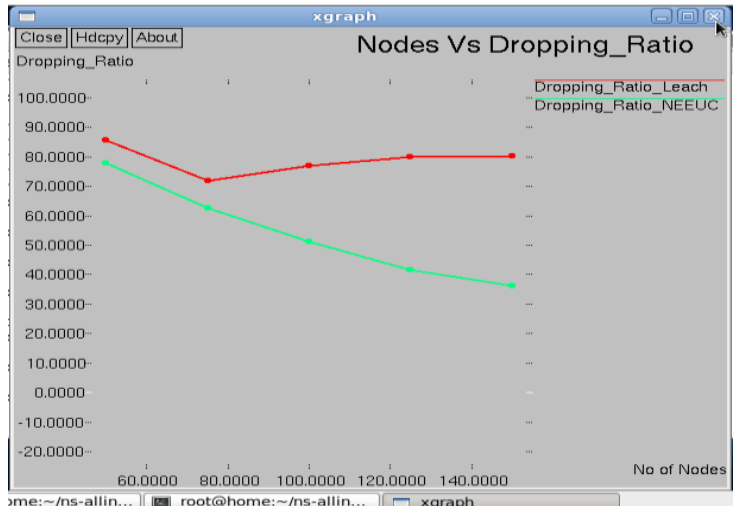

Fig. 7 Node vs Dropping Ratio

Fig 8 show the delay evaluated against the variation in the number of nodes with the senders. The delays include all the delays that are occurred by buffering during route discovery latency, retransmission delays. The delay of NEEUC is reduced by 1 to 3 milliseconds when compared with LEACH

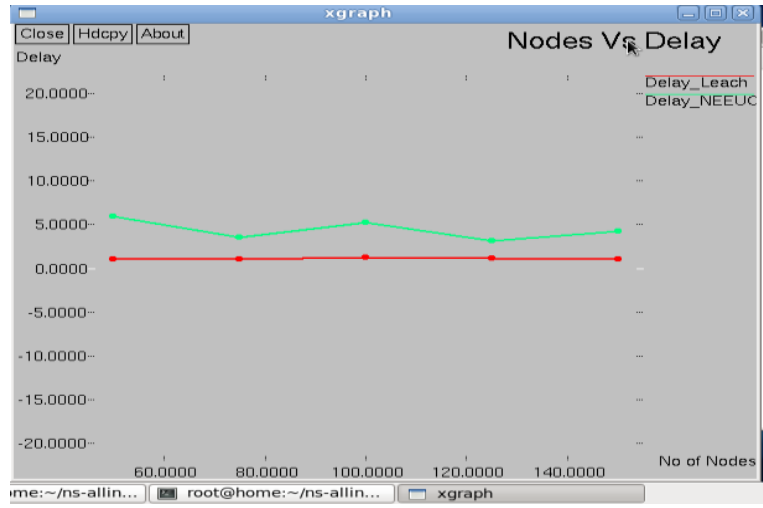

Fig. 8 Node Vs Delay Ratio

In fig 9 the average energy in NEEUC is better than LEACH and EEUC

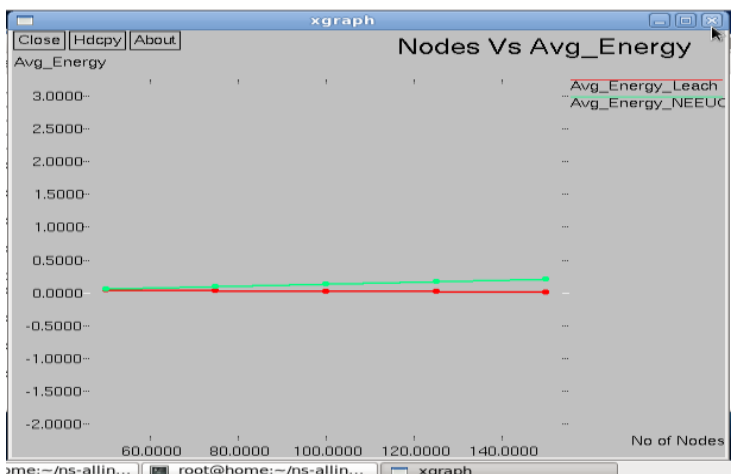

Fig 9 Node vs Average Energy

\section{CONCLUSION}

In this paper, we have introduced a novel unequal clustering routing protocol for wireless sensor networks. The hot spot problem arises when employing the multi hop routing in a clustered sensor network. To address the 


\section{International Journal of Advanced Research in Computer and Communication Engineering} ISO 3297:2007 Certified

Vol. 5, Issue 12, December 2016

hotspot problem, we first introduce an unequal clustering As well as we introduced multi hop routing. Cluster heads closer to the base station have smaller cluster sizes than those farther from the base station, thus they can preserve some energy for the purpose of inter-cluster data forwarding unequal layer inter clustering.

\section{REFERENCES}

[1] Wendi Rabiner Heinzelman, Anantha Chandrakasan, Hari Balakrishnan, "Energy-Efficient Communication Protocol for Wireless Microsensor Networks", Proceedings of the 33'd Hawaii International Conference on System Sciences, 2000

[2] Liu Pin, Huang Ting,Zhou Xiou etl," An Improved Energy efficient Unequal Clustering Algorithm of Wireless sensor Network", IEEE 2010 .

[3] Ossama Younis, Sonia Fahmy, "HEED: A Hybrid, EnergyEfficient, Distributed Clustering Approach for Ad Hoc Sensor Networks", IEEE Transaction on Mobile Computing,Vo!.3, No.3, 2004, pp366-349

[4] LiCF, YeM, Chen G H, etaI, "An energy-efficient unequal clustering mechanism for wireless sensor networks", Proceedings of the 2nd IEEE International Conference on Mobile Adhoc and Sensor Systems (MASS 2005), Washington , DC ,2005[C] .

[5] I.F. Akyildiz, W. Su, Y. Sankarasubramaniam and E. Cayirci, "A survey on sensor networks", IEEE Communications Magazine 40 (August 2002) 102-114.

[6] Mainwaring, J. Polastre, R. Szewczyk, D. Culler and J. Anderson,'Wireless sensor networks for habitat monitoring", Proceedings of ACM Workshop on Wireless Sensor Networks and publications (September 2002) pp. 88-97.

[7] Krishnamachari, D. Estrin and S. Wicker," The impact of data aggregation in wireless sensor networks, ",Proceedings of IEEE International Conference on Distributed Computing Systems Workshops (July 2002) pp. 575-578.

[8] W. Heinzelman, A. Chandrakasan and H. Balakrishnan, "Energy efficient communication protocols for wireless microsensor networks", Proceedings of the 33rd Hawaiian International Conference on Systems Sci ence (January 2000).

[9] V. Mhatre and C. Rosenberg, "Design guidelines for wireless sensor networks: communication, clustering and aggregation", Ad Hoc Networks 2(1) (2004) 45-63.

[10] K. Akkaya and M. Younis, "A survey on routing protocols for wireless sensor networks", Ad Hoc Networks 3(3) (2005) 325-349.

[11] W. Heinzelman, A. Chandrakasan and H. Balakrishnan,"An application-specific protocol architecture for wireless micro sensor networks", IEEE Transactions on Wireless Communications 1(4) (2005) 660-670. Springer Wireless Net.

[12] W. Choi, P. Shah and S.K. Das," A framework for energy-saving data gathering using two-phase clustering in wireless sensor networks", Proceedings of International Conference on Mobile and Ubiquitous Systems (August 2004) pp. 203-212.

[13] O. Younis and S. Fahmy, "HEED: a hybrid, energy-efficient, distributed clustering approach for ad hoc sensor networks", IEEE Transactions on Mobile Computing 3(4) (2004) 660-669.

[14] M. Qin and R. Zimmermann, "An energy-efficient voting based clustering algorithm for sensor networks", Proceedings of $1^{\text {st }}$ ACIS Workshop on Self-AssemblingWireless Networks (May 2005).

[15] J.S. Liu and C.H. Lin," Energy-efficiency clustering protocol in wireless sensor networks", Ad Hoc Networks 3(3) (2005) 371-388.

[16] M. Ye, C. Li, G. Chen and J. Wu," An energy efficient clustering scheme in wireless sensor networks", Ad Hoc \& Sensor Wireless Networks to appear.

[17] S. Soro and W. Heinzelman, "Prolonging the lifetime of wireless sensor networks via unequal clustering", Proceedings of the 19th IEEE International Parallel and Distributed Processing Symposium (IPDPS) (April 2005).

[18] T. Shu, M. Krunz and S. Vrudhula, "Power balanced coverage-time optimization for clustered wireless sensor networks," Proceedings of the Sixth ACM International Symposium on Mobile Ad Hoc Networking and Computing (MobiHoc) (May 2005).
[19] M. Perillo, Z. Cheng andW. Heinzleman, "An analysis of strategies for mitigating the sensor network hot spot problem,"Proceedings of the Second International Conference on Mobile and Ubiquitous Systems (July 2005).

[20] S. Olariu and I. Stojmenovic," Design guidelines for maximizing lifetime and avoiding energy holes in sensor networks with uniform distribution and uniform reporting," Proceedings of IEEE INFOCOM (April 2006).

[21] J. Lian, K. Naik and G. Agnew, "Data capacity improvement of wireless sensor networks using non-uniform sensor distribution", International Journal of Distributed Sensor Networks to appear.

[22] E.I. Oyman and C. Ersoy," Multiple sink network design problem in large scale wireless sensor networks, "Proceedings of the International Conference on Communications (June 2004).

[23] Ashanie Gunathillake and Kithsiri "An unequal clustering Algorithm for an Emergency Response Wireless Sensor Network" IEEE 9th International conference on Mobile adhoc \& sensor network, 2013.

\section{BIOGRAPHIES}

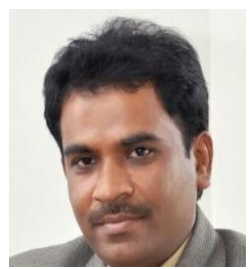

Prof. N. R. Wankhade completed his post-graduation from Bharti Vidyapith, Pune, Maharashtra. Presently he is working at LGN Sapkal college of engineering, Nashik, Maharashtra, India as a professor and head of computer engineering department. He has presented papers at National and International conferences and also published paper in national and international journals on various aspect of the computer engineering and WSNs. His research of interest include computer networks, network security, wireless sensor network.

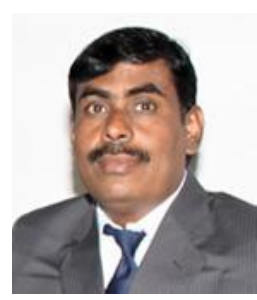

Dr. Dinesh $\mathbf{N}$ Chaudhari, is working as Professor and Dean in computer Engineering department of Jawaharlal Darda Institute of Engineering \& Technology, Yavatmal. He is recognized Ph.D. guide at Amravati University and has more than 20 years of academic experience. His interests are in cloud computing, computer networking and security; and he has written many papers in national/international Conferences and journals. 